\title{
Research on Diffusion Strategy About Resource Index of MP2P
}

\author{
Fangfang Guo, Jing Xu
}

Department of Computer Science and Technology, Harbin Engineering University, Harbin, China

\begin{abstract}
The inherent mobility of nodes leads to frequent changes in network structure and resource location delay larger in MP2P network. Existing search algorithms for MP2P is not efficient enough, and is only for single application model. In this paper an epidemic diffusion strategy about index of resource is proposed to implement diffusion resources to solve low efficiency and long latency positioning defects in a large search. Results demonstrate the principle of the proposed algorithm can improve the resource search efficiency.
\end{abstract}

Index Terms: MP2P; resource index; epidemic

(C) 2012 Published by MECS Publisher. Selection and/or peer review under responsibility of the Research Association of Modern Education and Computer Science

\section{Introduction}

MP2P as the integration of mobile technology and P2P network model is an important de velopment in the application direction the future wireless networks. MP2P based on P2P fully distributed network technology and mobile network, has good scalability, robustness, cost-effective, flow balancing, and low cost of selforganization, and also adapt to the user's request of connection to the network at any time. However, due to the inherent mobility of MP2P nodes, it leads to a lot of technologies, which are relatively mature in p2p, can't be directly applied in MP2P, such as: topology changes frequently, no unified network layer routing algorithms, long delay and so on. [1,2] Today the design of search algorithm in MP2P is an important basic research. But most researchers focus on the design of search algorithms in specific model, they are not good solutions to mobility and positioning accuracy. This paper presents the diffusion index of resources in the direction of the resource providers, thereby improving the efficiency of resource location.

\section{Relation Work}

Search of resources concentrated in the resource representation and resource search algorithm. Common methods of resources that mainly include the index method of using of resources, compress the bulk of the resource data, which reduces the amount of data transfer information. Small amount of index data resources, light query algorithm, is a good carrier of the resource request algorithm. Researchers study the index of

* Corresponding author.

E-mail address: guofangfang@hrbeu.edu.cn, xujing006@hrbeu.edu.cn 
resources focused on the index data structure, indexes the contents of the organization and manner of publication of the index three. One index the order of the index data structure often is divided into: inverted index [3], the index tree $[4,5]$ and distributed indexing [6].The order of the index search algorithm is simple but a longer delay, inverted index and the index tree data structure using a hierarchical index find the index of the time reduction, subject to the introduction of buffer space. Distributed index be satisfied with reducing the time and space requirements, but the network structure is fit for distributed. The article used the distributed index structure in conjunction with the tree-index method.

Most resource search algorithm is fit for a particular MP2P architecture. Reference [7] investigates finding and sharing of resources of the social network of the cell phone. The proposed query mechanism allows mobile users communicate with each other for resource located on three criteria: the limited depth of inquiry; avoid redundancy query; known path query. Although to some extent its mobile customers to solve the transfer request message, but the drawback is an increase in the request packet path information query mechanisms to put the network will increase the amount of data, limited bandwidth, large-scale mobile communication networks. It is availability to be studied further. JiuJun Cheng in Beijing posts and telecommunications university put forward a simple and effective search mechanism for the index server [8].The results showed that, comparing searching method with the index server proposed and flooding search mechanism for mobile P2P systems, it reduces the energy cost to make up for the lack of mobile terminals. But when the resource request nodes and destination nodes are in different cellular networks, the repeated requests for the same resources go through the index server every time that will bring great delay and unnecessary bandwidth overhead.

\section{Resources Index Release}

In this paper, the structural model is a half distributed architecture model with super-node that mainly researchers accepted. [9] Ordinary node layer is distributed network and registered to the super-nodes, so stored the index tree in the super-nodes. Backbone network is structured networking, so using a distributed index structure.

\section{A. The Contents of the Index of Resources}

Resource content of the resource index is the data structure identified, includes resources for key (RKEY), resource identifier and resource location information stored. The content of FID is shown in Fig 1.

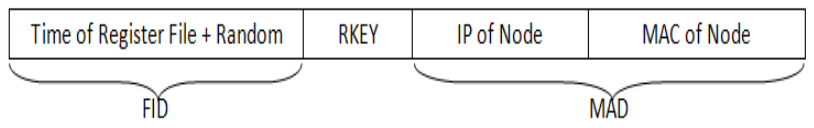

Figure 1 The content of index

\section{B. Organization of Resource Index}

Resource index stores in different layers of the network. When resource location, finding in resource index, the contents of index can be used to targeted to the node that storage resources, so as to completing the process of positioning resources. Index stored in ordinary node in line in according with the resources to retrieve the number of reverse order.

\section{The Diffusion Of resource index}

The fundamental characteristics of MP2P are fully distributed network model without central control, and the dynamic change is strong. Release its index to the network is an effective means to reduced energy consumption and improves the positioning effects. Diffusion is an important way to increase the amount of information resources in network. BY the diffusion of resources, resource requests can effectively improve the corresponding speed and reduce the search delay. Taking into the large amount of information resources and data, spreading all 
the data to the network is not realistic and will generate a lot of redundant data, leading to the collapse of the network, so take use of releasing index of resources.

\section{Diffusion Index}

Epidemic model as a mathematical research model is a good application prospects in this unstructured distributed P2P network. Epidemic model has three categories nodes: S class (Susceptible), refers to the nodes who are not infected, but are infection source of the objects; I class (Infective), refers to the objects with infectious disease. It can spread diseases to the S class members; R class (Removal), refers to the objects those are isolated or not infected objects. There are many epidemic models. Typical ones include SIR (susceptible infected removed) $[10,11]$ and the SIS (susceptible Infected susceptible) $[12,13]$. SIS model mainly considers the objective state of the infected objects, emphasizing the infectious process. But transmission node statues maintenances are ill-considered. SIR takes into all possible statues of nodes in the transmission process, but is poor ability in the maintenance of the network link. Consideration to the advantages and disadvantages between the two network models, the article decides to use the SIRS model (short-term immunity removed susceptible) [14].

As the disease spread in complex networks is equal probability distribution, the transition between three states as shown in Fig2, we get:

$\mathrm{SN}(\mathrm{t})+\mathrm{IN}(\mathrm{t})+\mathrm{RN}(\mathrm{t})=1$

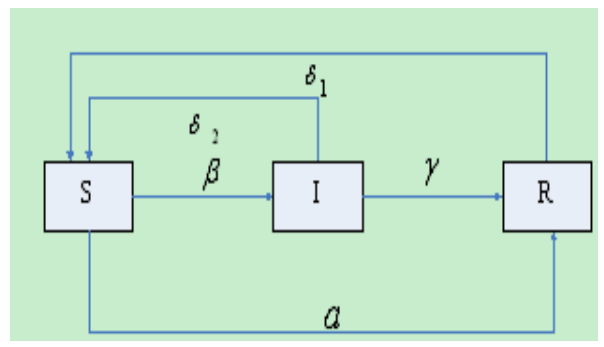

Figure 2 State transition

SIRS, in combination with the advantages of the SIS and the SIR, uses short-term immunity, making the removal nodes in the secondary infection to ensure the effectiveness and continuity of resources diffusion path. Network nodes are in 3 categories: susceptible node (SN), the infected nodes (IN), recovered node (RN). SN: refers to the nodes in the transmission path which are not infected but infective. It can diffuse resource index.

- IN: refers to the nodes that received spread of the index. It can diffuse index to the SN class members;

- $\quad \mathrm{RN}$ : refers to the nodes that have received the contents of spread index. This class is now only forwards the message, not backed up and diffusion.

SN (t), IN (t), RN (t) mean the probability of node in three different states at time t. $\alpha, \beta, \gamma, \delta 1, \delta 2$ mean the probability of conversion between the three states.

\section{Implementation of Diffusion}

In the SIRS model, each node in the process of joining the network is in the state of SN, in order to complete the network of the communication path establishing, maintaining the continuity of the network channel. When the ordinary nodes push its own resource index into the network, it initialized its concentration of resource request (CRR), download diffusion function of epidemic, and the initialize trigger threshold (TT) of epidemic function.

- $\quad \mathrm{CRR}=$ times of resources visited / times of node visited.

- EDF: diffusion algorithm designed from epidemic algorithm, mainly about diffusion direction and definition the extent.

- TT: the threshold of trigger diffusion function, controlling the amount of diffusion data. 
With the node activities, the amount of request increasing, then ultimately is up to TT of EDF to begin the diffusion of resources. Write the resource indexes that reach the TT to the proliferation table. EDF is used to spread the information to other nodes in the SN status. The algorithm uses the spread hops are 7. Diffusion algorithm is shown in Fig 3. TT can avoid too much amount of proliferation data so as to waste of network bandwidth resources, increase network load and lead to network collapse.

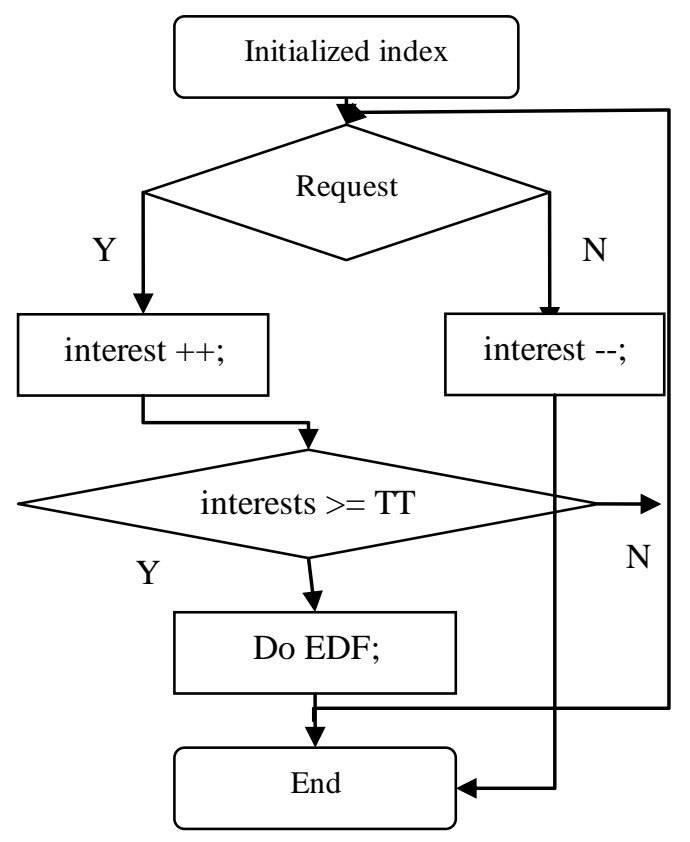

Figure 3. Diffusion Process

\section{E. Maintain the Proliferation Resource Index}

The network will appear a lot of resource index back up after diffusion. It can undoubtedly increase positioning speed and reduce the positioning time delay. However, it will bring a lot of redundant data to the network, and network load increase exponentially. Diffusion indexes received storage in independent resource index table. TTL is greater than 0. Diffusion resource index table refreshes on time. When there is no request for a certain index, the TTL of the index minus one, and vice versa plus one. The index with spread information will be deleted when survival time is not greater than 0 .

In epidemic model, the node in the state of RN can't continue to disseminate information. To ensure the channel connectivity, the article uses short immune status to keep the contradiction balance between the Characteristics of MP2P and an epidemic model. When the mobility of nodes damaged stability of network link the node is set to state set R, enable short-term immunization (SI), thus avoiding the instantaneous topology changes frequently. It is used to maintain the network connectivity and channel stability.

\section{Simulations}

In order to verify the efficiency of the algorithm, we spread from two aspects of the success rate of the resource positioning and the network load is proved. The test uses NS2 platform to validate and evaluate the proposed method. Mobile nodes randomly distributed in the $800 \mathrm{~m} \times 800 \mathrm{~m}$ rectangular area within the coverage area of nodes are $250 \mathrm{~m}$, bandwidth of $2 \mathrm{Mb} / \mathrm{s}$. In the test there are a total of 600 different size resources randomly distributed in the mobile nodes, the transmission radius is set to 7 hops. The node use Random 
Waypoint mobility model (Based on the random path of ad hoc network mobile model, the mobile node speed, direction and purpose are independent randomly selected), the pause time is 20s, simulation time is set to 1000s.Mobile node issued resources positioning news time at intervals between the $3 \mathrm{~s} \sim 10 \mathrm{~s}$.

\section{F. Success Rate of Localizations}

The success rate of localization can determine the publishing method to join diffusion mechanism significantly improved the application efficiency of the network. The results compared the difference between the resource localization method with index diffusion mechanism and without using index diffusion mechanism of resources positioning success rate. Resource request for the request algorithm using random walk search algorithm, using $\mathrm{K}=7$, K Road random forwarding the request. The results are shown in Fig 4 . In the Fig 4 we can see the introduction of the diffusion mechanism source index $\mathrm{K}$ road random forwarding positioning can well improve localization success rate, positioning it to the high success rate close to flooding search methods.

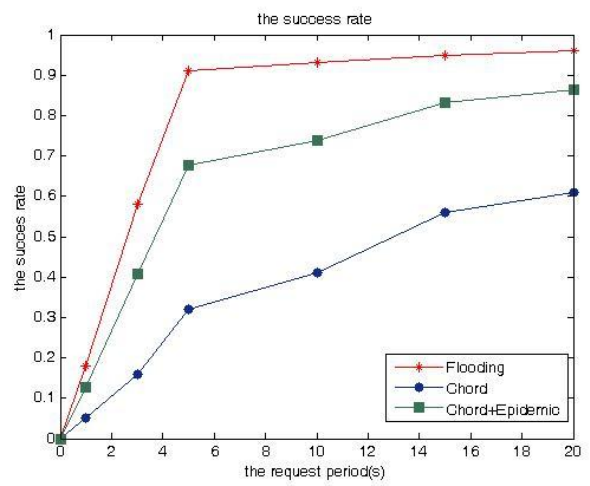

Figure 4. Positioning success rate

\section{G. Bandwidth and Loads}

Because MP2P node's ability is limited, mobile interconnection wireless channel bandwidth constraints, frequency instability, so they requested resource locator of the localization algorithm used lightweight as possible to reduce energy consumption, make the mobile nodes can be more long-term in working condition. Experimental results of three methods in comparative can meet the needs of the premise of the efficiency of resource location, network load changes. Fig 5 shows the test results, the longer request time interval, the lower the network load, network, the less number of messages delivered. Compare three search algorithms we can know: flood query method has the largest network load flow, distributed Chord algorithm produces lowest network load.This paper presents a method with the spread of infectious diseases, K Road, random walk method, the diffusion of making the network load much more than general random search method, but it will not produce excessive amount of information that not be tolerated.

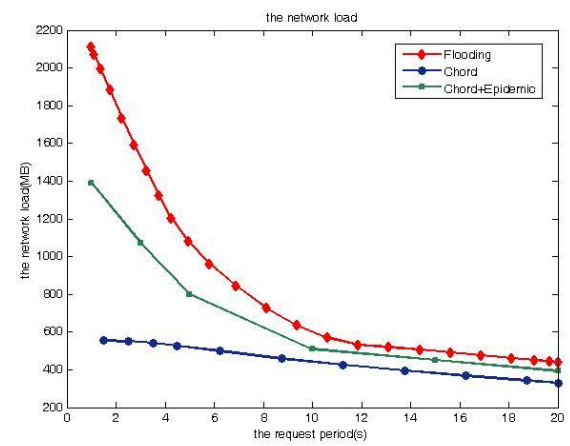

Figure 5. network load 


\section{Conclusions}

The key of MP2P network resources index release is index effective maintenance and resources diffusion, thus ensuring the efficiency of resource release, providing strong support resources accurate positioning and transmission .The method this paper put forward can be used on whole distributed mobile terminal, universality, regarding the network hierarchy no property requirements, can satisfy the requirements of MP2P resource sharing. The release and the search of resources is a pair of reciprocal and mutually supporting technology, so the key of future work is more focus on lightweight and specific requirements of weak search algorithm, making MP2P can better applied in real life.

\section{Acknowledgment}

This work was supported, in part, by the Key project of the 11th Five-Year Plan of the National Defences Pre-Research Foundation (Grant No. 513XXX602), by Scientific and Technological Project of Heilongjiang Province (Grant No. GC09A104), by the fund of Harbin (Grant No. 2010RFQXG026), by the Fundamental Research Funds for the Central Universities, and by National Natural Science Foundation important project(90718003).

\section{References}

[1] HK Zhang, Mobile Internet technology, Present and Future, Communications Development Forum, 2004

[2] C. Zhang, X. "Qiu.Comprehensive Analysis of P2P Technology[M]". People Press .March 2010, pp.371-373

[3] B. Nam ,A. SussmanSpatial. "Indexing of Distributed Multidimensional Datasets", 2005 IEEE International Symposium on Cluster Computing and the Grid,2005,pp.743-750

[4] M. Asadi, A. Haghighat, "A Scalable lookup Service for P2P File Sharing in MANET”, IWCMC'07, Honolulu, Hawaii, USA, August 12-16, 2007, pp.511-516.

[5] G. Peng, S. Li, "M-CAN: a Lookup Protocol for Mobile Peer-to-Peer Environment”,Proceedings of the 7th International Symposium on Parallel Architectures, Algorithms and Networks[C],[S.l.],[s.n.],2004.

[6] Hofstatter Q, Zols S, Michel M, et al. "Chordella-A Hierarchical Peer-to-peer Overlay Implementation for Heterogeneous, Mobile Environments [C] ". The Eighth International Conference on Peer-to-Peer Computing(P2P'08). Aachen, Germany:IEEE,2008:75-76.

[7] BAE J,LEE S,JUNG E,et al.A Mobile Peer-to-Peer Query in a Social Network. International Conference on Advanced Language Processing and Web Information Technology,Dalian Liaoning,China,July,2008:450-453P

[8] JJ Cheng, YH Li, SR Cheng, Research on mobile P2P system architecture and key technologies,Beijing University of Posts and Telecommunications.2006,29(4), pp.86-89

[9] M. Papadopouli, H. Schulzrinne, Seven. "Degrees of Separation in Mobile AD Hoc Networks[C]"//IEEE Global Telecommunications Conference,San Francisco, US:IEEE, 2002,pp.1707-1711.

[10]X. Z. Li, W.S. Li, Mini Ghosh. Stability and bifurcation of an SIR epidemic model with nonlinear incidence and treatment. Applied Mathematics and Computation; 2009, 210:141-150.

[11]S. R. He, H. Hai. Using content 1.5 to analyze an SIR model for childhood infectious diseases. Communications in Nonlinear Science and Numerical Simulation; 2008, 13:1743-1747.

[12] X. Zhang, X. N. Liu. Backward bifurcation and global dynamics of an SIS epidemic model with general incidence rate and treatment. Nonlinear Analysis; 2009, 10:565-575.

[13]H. J. Shi, Z. S. Duan, G. R. Chen. An SIS model with infective medium on complex networks. Physic A; 2008, 387:2133-44.

[14] T.L Zhang, Z. D. Teng. Permanence and extinction fo a no autonomous SIRS epidemic model with time delay. Applied Mathematical Modelling; 2009, 33:1058-1071. 